\title{
Tolerable Duration of Warm Ischaemia After Circulatory Death Is Safe For At Least One Hour in Porcine Lungs: Functional Assessment with Ex Vivo Lung Perfusion
}

\author{
Mathilde M. B. Hebsgaard, MD,${ }^{1}$ Hannelouise Kissow, $\mathrm{MD}, \mathrm{PhD},{ }^{2}$ Nikolaj B. Lilleør, RC, ${ }^{1}$ \\ Ulver S. Lorenzen, MD ${ }^{1}$ Raphaelle A. Chemtob, MD ${ }^{3}$ Hasse Møller-Sørensen, MD, ${ }^{4}$ \\ Peter S. Olsen, MD, PhD, ${ }^{1}$ Christian H. Møller, MD, $\mathrm{PhD}^{1,5}$ \\ ${ }^{1}$ Department of Cardiothoracic Surgery, Copenhagen University Hospital, University of Copenhagen, Denmark; \\ ${ }^{2}$ NNF Center of Basic Metabolic Research and Department of Biomedical Sciences, University of Copenhagen, Denmark; \\ ${ }^{3}$ Department of Thoracic and Cardiovascular Surgery, Heart and Vascular Institute, Cleveland Clinic, Cleveland, Ohio, USA; \\ ${ }^{4}$ Department of Cardiothoracic Anaesthesiology, Rigshospitalet, University of Copenhagen, Denmark; 5Department of Clinical \\ Medicine, University of Copenhagen, Copenhagen, Denmark
}

\section{ABSTRACT}

Background: Lungs from donation after circulatory death (DCD) may be an underused resource for transplantation. The aim was to investigate, with a DCD pig model, if it was possible to recondition lungs exposed for up to $2 \mathrm{~h}$ of warm ischaemia with ex vivo lung perfusion (EVLP).

Methods: Danish domestic pigs $(N=17)$ were randomized into three groups. In the two study groups, lungs were exposed to either 1 or $2 \mathrm{~h}$ of warm ischaemia. All lungs were reconditioned and evaluated after $83 \pm 38$ minutes of perfusion at $\mathrm{FiO}_{2} 1.0$ and 0.21 with EVLP. Outcome measures were gas exchange, pulmonary physiology, inflammatory markers, and histopathologic assessment score.

Results: Lungs exposed for $2 \mathrm{~h}$ of warm ischaemia did not meet the criteria: $\mathrm{PaO}_{2}>13 \mathrm{kPa}$ required for donation compared with lungs subjected to 0 and $1 \mathrm{~h}$ of warm ischaemia $(11.0 \mathrm{kPa}$ vs. $14.2 \mathrm{kPa}, P<0.001)$. These lungs also developed an increased amount of foam and fluid in the airways. No differences in $\mathrm{PaCO}_{2}$, compliance, or pulmonary vascular resistance were observed.

Conclusion: Results show that while lungs subjected to 0 or $1 \mathrm{~h}$ of warm ischaemia meet the criteria for transplantation based on EVLP evaluation, lungs subjected to $2 \mathrm{~h}$ of warm ischaemia did not.

\section{INTRODUCTION}

Donor organ shortage is still a limiting factor in lung transplantation, and the number of patients on waiting lists are increasing [Boffini 2015]. In most transplant centers, donor

Received October 14, 2021; accepted November 2, 2021.

Correspondence: Matbilde Marie Broensro Hebsgaard, Sankt foergensgade 11, 9- 3, 9000 Aalborg, +45 61468801 (e-mail: Hebsgaard27@hotmail.com). organs come exclusively from brain death donors (BDD) [Botha 2008]. However, a few centers have taken up donation after circulatory death (DCD) in an effort to increase the pool of available organs.

There is reluctance to use DCD lungs due to warm ischaemia following circulatory arrest and the risk of primary graft dysfunction [Porteous 2017]. In some centers, transplantation using DCD lungs in the Maastricht Category III [Kootstra 1995] have been done with long-term outcomes similar to transplantation with lungs from BDD [Van Suylen 2017]. Donor organs belonging to Maastricht Category III are subject to a short duration of warm ischaemia, since withdrawal of life-sustaining therapy and retrieval of organs is carefully planned. In the uncontrolled DCD setting, warm ischaemia often is longer and the exact duration is often unknown [Inci 2017]. However, utilization of DCD lungs is low and only performed in a few countries [Inci 2017]. The ex vivo lung perfusion (EVLP) technique was developed over the last decade, thereby creating possibilities for evaluation and reconditioning of donor lungs [Machuca 2013].

In this study, we use EVLP as a translational research platform to investigate the consequences of increasing durations of warm ischaemia on lungs from circulatory death pigs in an animal experimental setting.

\section{MATERIAL AND METHODS}

The study was conducted with permission from the Danish animal experiments inspectorate (License No. 201515-0201-00573). All animals received human care, as per the European Convention for the Protection of Vertebrate Animals used for Experimental and Other Scientific Purposes, Guide for the Care and Use of Laboratory Animals, as well as local institutional guidelines.

The experimental set up was conducted at the Department of Experimental Medicine at the University of Copenhagen. We enrolled 17 Danish female domestic pigs with a mean mass of $59 \mathrm{~kg}$ (range 51-63 kg). Heparin (20000 IE) was administered pre-mortem. 
The animals were randomized into three groups, including a control group in which cardiac arrest was induced and the lungs immediately were procured. In the two intervention groups, cardiac arrest was induced, and the animals were left untouched for 1 or $2 \mathrm{~h}$ of warm ischaemia before the lungs were harvested.

Cardiac arrest by ventricular fibrillation was induced with a direct current (9-voltage battery), introduced to the pericardium through the diaphragm to reach the apex of the heart. The pig was left untouched for 1 or $2 \mathrm{~h}$ of warm ischaemia, depending on the randomization protocol. At induction of ventricular fibrillation, the lungs were inflated to a peak pressure of $30 \mathrm{cmH} 2 \mathrm{O}$, with the tube clamped during warm ischaemia.

Approximately 20 min before warm ischaemia was ended, a median sternotomy was performed, exposing the heart and pulmonary artery. After this, the lungs were flushed anterograde via the pulmonary artery with 1 liter of $4^{\circ} \mathrm{C}$ of preservation fluid (Custodiol ${ }^{\circledR}$ HTK Solutions, Essential Pharmaceuticals, LLC, Durham, NC, USA). A tip of the left atrial appendage was amputated after the pulmonary flush, and the heart-lung bloc was explanted.

In the control group, the sternotomy was performed, and the pulmonary artery was cannulated directly after the induction of cardiac arrest, and the lungs were immediately flushed.

We used the Vivoline LS1 EVLP system (Vivoline Medi$\mathrm{cal}^{\circledR}$, Lund, Sweden) for lung reconditioning and evaluation. The EVLP system was primed with $500 \mathrm{ml}$ of autologous washed red blood cells to a target haematocrit of $12-15 \%$ and a homemade mixture, similar in composition to the Steen Solution $^{\mathrm{TM}}$ (XVIVO Perfusion AB, Göteborg, Sweden). Subsequently, $20 \mathrm{ml}$ of addex-THAM $(3.3 \mathrm{mmol} / \mathrm{ml}$, Fresenius Kabi AB, Uppsala, Sweden) was added to the priming solution, and in addition to this, $1 \mathrm{ml}$ per $-1 \mathrm{HCO}_{3}$ - until the base excess was between -5 and 5 .

The lungs were connected to the EVLP and perfusion through the pulmonary artery was started with a flow of $1.5 \mathrm{l} /$ min. Flow was slowly increased, aiming for $4.0 \mathrm{~L} / \mathrm{min}$ while the lungs were rewarmed. At $32^{\circ} \mathrm{C}$, ventilation of the lungs was started; initially, with low tidal volumes $(2-3 \mathrm{ml} / \mathrm{kg})$ and $\mathrm{FiO}_{2} 0.50$, which was slowly increased to a target tidal volume of 6-8 $\mathrm{ml} / \mathrm{kg}$, PEEP $5 \mathrm{cmH}_{2} \mathrm{O}$, and a frequency of $12 / \mathrm{min}$.

At $36^{\circ} \mathrm{C}$, an arterial blood gas analysis from mixed arterial blood from all four pulmonary veins was assessed. At $\mathrm{PaO}_{2}>$ $40 \mathrm{kPa}$, the EVLP system was shifted to an evaluation mode. In this phase, the oxygen supply was disconnected from the oxygenator. The lungs were ventilated with $\mathrm{FiO}_{2} 1.00$ for 10 min, then $\mathrm{FiO}_{2}$ was reduced to 0.21 for $10 \mathrm{~min}$. After each 10-min interval, $\mathrm{PaO}_{2}$ was assessed by an arterial blood gas analysis from the pulmonary veins. Acceptable oxygenation for transplantation was $\mathrm{PaO}_{2}$ with $>50 \mathrm{kPa}$ at $\mathrm{FiO}_{2} 1.00$ and $>13 \mathrm{kPa}$ at $\mathrm{FiO}_{2} 0.21$.

Samples for arterial gasses were drawn from the carotid artery before induction of ventricular fibrillation, and from the pulmonary veins during EVLP. Before EVLP was initiated, biopsies were obtained from the right apical lung lobe. Perfusion buffer samples were taken from pulmonary veins 5 min after the start of EVLP (pre-EVLP) and repeated at the end of the experiment (post-EVLP). Biopsies were fixed in $4 \%$ paraformaldehyde for histological analysis or were snap frozen and stored at $-80^{\circ} \mathrm{C}$ until analysis. The bronchoalveolar lavage (BAL) fluid and perfusion buffer were transferred to ice-cold EDTA tubes and centrifuged $\left(15 \mathrm{~min}, 1600 \mathrm{G}, 4^{\circ} \mathrm{C}\right)$. Supernatant was maintained in a frozen state $\left(-20^{\circ} \mathrm{C}\right)$.

The primary outcome was lung function as assessed by oxygenation capacity, pulmonary vascular resistance (PVR), and dynamic lung compliance at $\mathrm{FiO}_{2} 1.00$ and 0.21 .

Two biopsies of the same size from each time point $\left(\mathrm{T}_{0}\right.$ and $\mathrm{T}_{\text {end }}$ ) were analyzed for myeloperoxidase (MPO) activity, as previously described [Hytting-Andreasen 2018]. Absorbance change was measured at $450 \mathrm{~nm}$ in a mixture of supernatants and substrate buffer. Results were given as a mean of the two biopsies from each time point. For measuring IL-8 in lung tissue, biopsies were weighed and homogenized. Supernatants from tissue homogenate, BAL and perfusion buffer were assayed for porcine IL-8 (ELISA kit LS-F5330-1 LifeSpan BioSciences, Seattle, WA, USA), following manufacturer's instructions. Porcine TNF- $\alpha$ was measured in perfusion samples (ELISA kit EKP318 Nordic Biosite, Taby, Sweden).

Fixed tissue for histology was embedded in paraffin. Sections of $4 \mu \mathrm{m}$ were stained with haematoxylin and eosin, and four identifiable pathological parameters were scored on a scale of $0-4$, as previously described [Haam 2015]: (a) alveolar congestion, (b) hemorrhage, (c) leukocyte infiltration, and (d) thickness of the alveolar wall. A score of 0 represented normal lungs; 1) $<25 \%$ lung involvement; 2) $25-50 \%$ lung involvement; 3) 50-75\% lung involvement; and 4) > 75\% lung involvement. Scores were summed in order to reach a final total score. The origin of the samples was blinded to the observer.

Data are expressed as a mean with standard deviation or range. Groups were compared using a one-way analysis of variance (ANOVA) with a significance level at $P<0.05$. Statistics were performed using IBM SPSS Statistics v16.0 (SPSS Inc., Chicago, IL, USA).

\section{RESULTS}

No differences were found in animal mass, time from lung retrieval to EVLP start, or EVLP time between groups. (Table 1) Because of the set up, there were differences in warm ischaemic time between groups (Table 1).

Data from the EVLP can be seen in Table 2. (Table 2) During the EVLP evaluation phase, there were no significant differences in $\mathrm{PaCO}_{2}$, compliance, or PVR between groups at any $\mathrm{FiO}_{2}$ level. However, at $\mathrm{FiO}_{2} 0.21$, we observed a difference in $\mathrm{PaO}_{2}(P=0.001)$. In the $2 \mathrm{~h}$ warm ischaemia group, no lungs reached a $\mathrm{PaO}_{2}$ above $13 \mathrm{kPa}$. In comparison, all lungs in the control group, and all but one pair in the $1 \mathrm{~h}$ ischaemia group, reached the threshold. At $\mathrm{FiO}_{2} 1.0$, no difference in $\mathrm{PaO} 2$ was seen between groups, and all lungs reached a $\mathrm{PaO}_{2}$ $>50 \mathrm{kPa}$.

During EVLP, we observed a general increase in MPO activity, without a difference between groups. The IL-8 concentration in both tissue and the EVLP circulation did not 
Table 1. Donor animal characteristics

\begin{tabular}{|c|c|c|c|c|}
\hline Duration of warm ischaemia (min) (SD) & 0 & $61.3(60-65)$ & $119.8(119-120)$ & 0.003 \\
\hline EVLP-time $(\min )(+/-)$ & $78.8(60-90)$ & $84.8(62-122)$ & $86.3(62-122)$ & 0.789 \\
\hline$\Delta$ lung mass $(\mathrm{g})(+/-)$ & $85.8(-10-212)$ & $76.0(-3-174)$ & $82.6(-14-214)$ & 0.983 \\
\hline
\end{tabular}

Donor animal characteristics shown in means with standard deviation (duration of warm ischaemia) or with range (Time from retrieval to start EVLP, EVLP-time and $\Delta$ lung mass).

Table 2. EVLP Data

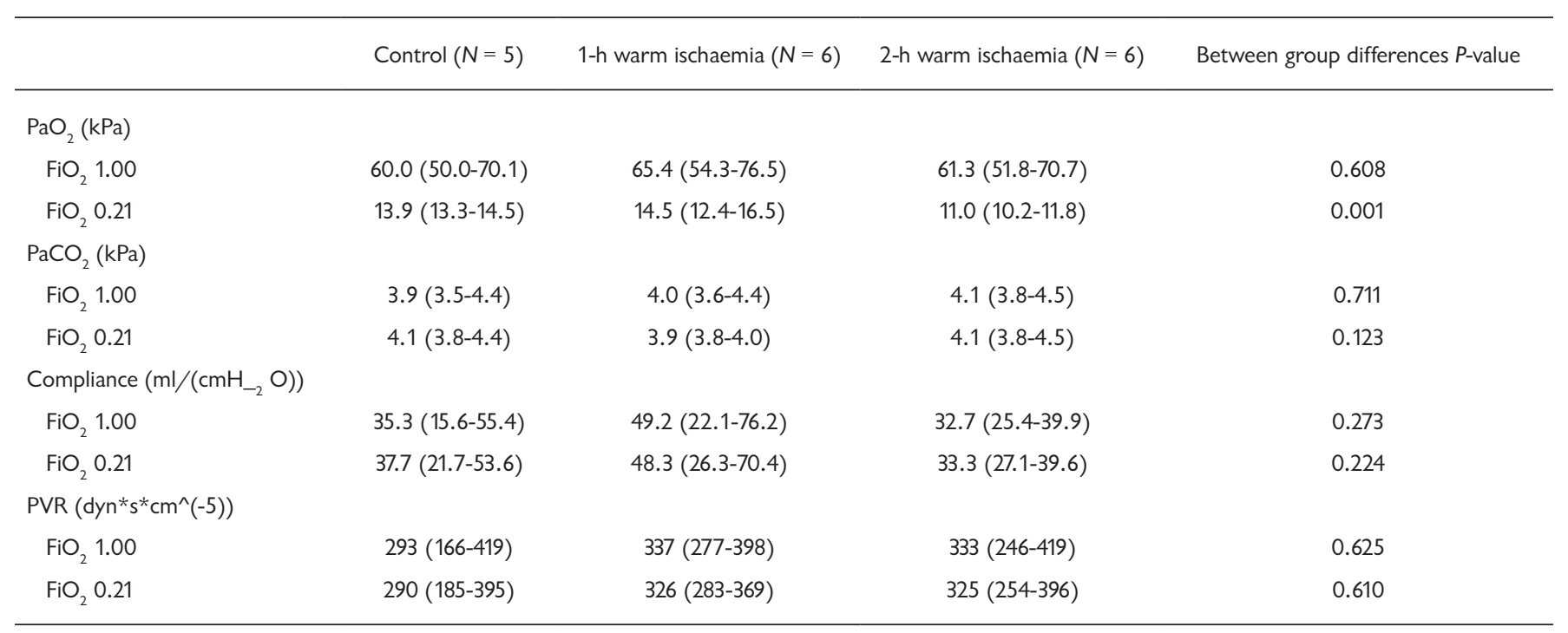

Data are expressed as means with range

differ between groups over time. (Table 3) TNF- $\alpha$ concentrations tended to increase in perfusion buffer during EVLP, which was significant in the $1 \mathrm{~h}$ group, but not in the control and $2 \mathrm{~h}$ groups. Levels of TNF- $\alpha$ in the perfusion buffer was higher in the 1 and $2 \mathrm{~h}$ ischaemia groups post-EVLP, in comparison with the control group $(P<0.005)$ (Table 3$)$.

Biopsies taken pre-EVLP in control pigs (no warm ischaemia) had a higher score than lungs exposed to 1 and $2 \mathrm{~h}$ of warm ischaemia $(P<0.05)$ (Table 3$)$. This was driven by alveolar congestion and thickness of the alveolar wall. At the end of the experiment (post-EVLP), the score in the control group had decreased to a level that was not different from the ischaemic groups (Table 3, Figure 1).

At the time of retrieval, lungs exposed to warm ischaemia showed gravitationally distributed hemorrhage and atelectasis, most pronounced in the $2 \mathrm{~h}$ ischaemia group. During EVLP, atelectasis was possible, but lungs exposed to $2 \mathrm{~h}$ of warm ischaemia developed foam and fluid in the airways. Post-EVLP, all lungs showed some degree of congestion, pronounced in the ischaemic groups and confirmed by 'the collapse test.' The majority of lungs gained mass after the

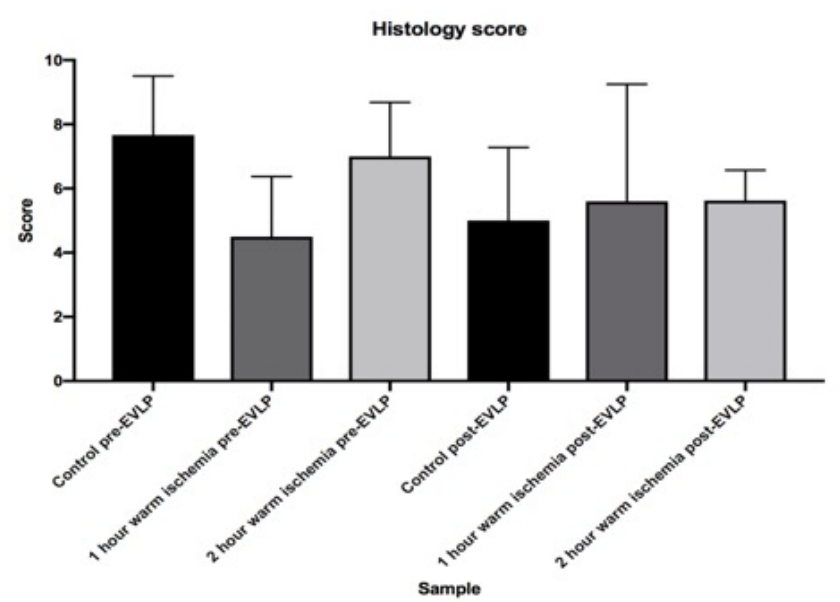

Figure 1: Diagram showing the change in histology scores after EVLP. This was lowered in all groups, especially the control group, in which the histology score was highest with post-warm ischaemia and pre-EVLP. 
Table 3. Inflammatory markers

Control $(N=5) \quad$ 1-h warm ischaemia $(N=6) \quad$ 2-h warm ischaemia $(N=6) \quad$ Between group differences $P$-value

\begin{tabular}{|c|c|c|c|c|}
\hline \multicolumn{5}{|c|}{ Histology score } \\
\hline Pre-EVLP & $8.2( \pm 1.2)$ & $4.5( \pm 1.9)$ & $7.0( \pm 1.6)$ & 0.020 \\
\hline Post-EVLP & $4.8( \pm 2.4)$ & $5.6( \pm 3.6)$ & $5.62( \pm 0.9)$ & 0.871 \\
\hline \multicolumn{5}{|c|}{ MPO activity in tissue (UMPO/biopsy) } \\
\hline Pre-EVLP & $594.0( \pm 191.7)$ & $378.0( \pm 256.7)$ & $604.0( \pm 452.4)$ & 0.433 \\
\hline Post-EVLP & $762.0( \pm 467.4)$ & $821.0( \pm 623.5)$ & $811.00( \pm 852.1)$ & 0.989 \\
\hline \multicolumn{5}{|c|}{ TNF- $\alpha$ - Perfusion buffer $(\rho \mathrm{g} / \mathrm{ml})$} \\
\hline Pre-EVLP & $2.2( \pm 2.9)$ & $16.7( \pm 19.1)$ & $5.4( \pm 8.6)$ & 0.160 \\
\hline Post-EVLP & $5.8( \pm 6.3)$ & $52.5( \pm 37.3)$ & $22.4( \pm 27.6)$ & 0.042 \\
\hline \multicolumn{5}{|c|}{ IL-8 - Perfusate (ng/ml) } \\
\hline Pre-EVLP & $3.4( \pm 3.5)$ & $6.2( \pm 2.7)$ & $5.4( \pm 4.5)$ & 0.462 \\
\hline Post-EVLP & $3.5( \pm 2.0)$ & $6.9( \pm 2.0)$ & $4.8( \pm 4.6)$ & 0.227 \\
\hline \multicolumn{5}{|c|}{ IL-8 - BAL (ng/ml) } \\
\hline Pre-EVLP & $0.2( \pm 0.3)$ & $0.7( \pm 0.9)$ & $0.5( \pm 0.8)$ & 0.521 \\
\hline Post-EVLP & $0.7( \pm 0.4)$ & $0.5( \pm 0.4)$ & $0.6( \pm 0.6)$ & 0.845 \\
\hline \multicolumn{5}{|c|}{ IL-8 - Biopsy (ng/ml) } \\
\hline Pre-EVLP & $1.7( \pm 0.8)$ & $1.7( \pm 0.6)$ & $1.9( \pm 2.5)$ & 0.952 \\
\hline Post-EVLP & $2.6( \pm 0.9)$ & $2.9( \pm 0.4)$ & $2.4( \pm 0.7)$ & 0.509 \\
\hline
\end{tabular}

First row: calculated histology scores in biopsies taken before and after EVLP. Second row: measured values of MPO activity. The rest is the measured values of inflammatory markers in biopsies, perfusate, and BAL-fluid. Data expressed as mean \pm SD.

EVLP run, with huge variation within groups, with no clear trend and no differences between them (Table 1).

\section{DISCUSSION}

In this study, we evaluated warm ischaemia in a DCD pig model. Lungs exposed to $2 \mathrm{~h}$ of warm ischaemia had a decrease in oxygenation capacity at $\mathrm{FiO}_{2} 0.21$ and did not reach the acceptance threshold for human transplantation. In contrast, at $\mathrm{FiO}_{2}$ 1.0, all lungs reached the threshold at $\mathrm{PaO}_{2}$ $>50 \mathrm{kPa}$. In the evaluation phase, the hemodynamics, $\mathrm{PaCO}_{2}$, and lung compliance of all lungs were acceptable, without differences between the three groups.

The first studies evaluating lungs exposed to warm ischaemia were performed on canines and rabbits [Egan 1991; Raemdonck 1998]. Based on these studies, the tolerable duration after circulatory arrest seemed limited to $1 \mathrm{~h}$. Yet, with the development of the modern ex vivo lung perfusion systems, renewed interest in extending the tolerable duration of warm ischaemia has evolved.

In a DCD pig model with prolonged blood-based normothermic EVLP, Spratt et al. found that lungs exposed to 1 and $2 \mathrm{~h}$ warm ischaemia showed improvement in PVR, mPAP (mean pulmonary artery pressure), and dynamic compliance after $24 \mathrm{~h}$ of EVLP. However, lungs exposed to $2 \mathrm{~h}$ of warm ischaemia showed reduced oxygenation capacity $(\mathrm{P} / \mathrm{F}$ ratio $<300 \mathrm{mmHg}$ with $\mathrm{FiO}_{2}$ 0.21) [Spratt 2017]. In contrast, Charles et al. concluded that warm ischaemic time could be extended up to $2 \mathrm{~h}$ in lungs exposed to warm ischaemia after hypoxic cardiac arrest, followed by $4 \mathrm{~h}$ of acellular normothermic EVLP [Charles 2018]. In this study, lungs subjected to 60 and $90 \mathrm{~min}$ of warm ischaemia had a P/F-ratio > 400 $\mathrm{mmHg}$ post EVLP at $\mathrm{FiO}_{2} 1.0$ and was accepted according to the Toronto criteria [Cypel 2011]. However, only 4 out of 6 lungs subjected to $120 \mathrm{~min}$ of warm ischaemia reached $\mathrm{P} / \mathrm{F}$-ratio $\geq 400 \mathrm{mmHg}$. Both studies coincided with our findings. At $\mathrm{FiO}_{2} 1.0$, we observed similar $\mathrm{PaO}_{2}$ between the 1 and $2 \mathrm{~h}$ warm ischaemia groups, but at $\mathrm{FiO}_{2}, 0.21$, impaired gas exchange was seen in lungs exposed to $2 \mathrm{~h}$ of warm ischaemia. Newly-transplanted lungs will be further exposed to ischaemia-reperfusion injury in the recipient, so we would be reluctant to conclude that lungs with acceptable oxygenation at $\mathrm{FiO}_{2} 1.0$, but marginal oxygenation at $\mathrm{FiO}_{2} 0.21$, should be transplantable.

Changes in inflammatory markers in a pig model during ischaemia may be difficult to interpret or compare to the reaction in human lungs. During the relatively short EVLP run in our study, we did not find any clear signal from the analysis of proinflammatory cytokines, TNF- $\alpha$ and IL-8, in the levels of MPO and leucocyte infiltration. This could be due to the short observation period of EVLP, which does not 
reveal long-term effects of acute ischaemic injury. In studies with $24 \mathrm{~h}$ EVLP and transplantation of donor lungs, a rise of IL-8 concentration, proportional to the duration of warm ischaemia, was observed [Charles 2018; Spratt 2017].

Our histopathological samples showed that control group lungs had increased alveolar congestion and thickness of the alveolar wall, indicating increased edema of the lungs compared with the 1 and $2 \mathrm{~h}$ groups. Hemorrhage and leucocyte infiltration levels were the same as in the other groups. After EVLP, total histopathological scores in the control group decreased, suggesting that EVLP may ameliorate congestion and edema in the lungs not subjected to warm ischaemia. However, lungs in the control group performed well on EVLP, regarding oxygenation capacity, indicating that baseline histological differences had no impact. As in previous studies, we could not demonstrate differences in the microscopic appearance between the control lungs and lungs exposed to warm ischaemia [Charles 2018; Spratt 2017; Spratt 2018].

There are a few limitations in this study. First, we only tested lungs with ex vivo lung perfusion, not by transplantation. However, lungs that did not improve and fulfill the criteria on EVLP would presumably not be transplantable. Second, the EVLP duration may have been too short to show changes in secondary outcomes. Finally, we administered heparin pre-mortem, which would be logistically and ethically difficult in some countries. On the other hand, one study shows that administration of heparin seems to not have crucial importance [Nilsson 2019].

To conclude, this study found that in a porcine animal experimental model, lungs subjected to $1 \mathrm{~h}$ of warm ischaemia showed acceptable criteria for transplantation. However, extending the warm ischaemic period to $2 \mathrm{~h}$ was associated with impaired oxygenation.

\section{REFERENCES}

Boffini M, Ricci D, Ranieri VM, et al. 2015. A bridge over troubled waters. Transplant int. 28(3):284-285.

Botha P, Rostron AJ, Fisher AJ, et al. 2008. Current Strategies in Donor Selection and Management. Semin Thorac Cardiovasc Surg. 20(2):143-151.

Charles EJ, Mehaffey JH, Huerter ME, et al. 2018. Ex vivo assessment of porcine donation after circulatory death lungs that undergo increasing Warm Ischemia times. Transpl Direct. 4(12):e405.
Cypel M, Yeung JC, Liu M, et al. 2011. Normothermic Ex Vivo Lung Perfusion in Clinical Lung Transplantation. N Engl J Med. 364(15):1431-1440

Egan TM, Lambert CJ, Reddick R, et al. 1991. A strategy to increase the donor pool: Use of cadaver lungs for transplantation. Ann Thorac Surg. 52(5):1113-1120.

Haam S, Lee S, Paik HC, et al. 2015. The effects of hydrogen gas inhalation during ex vivo lung perfusion on donor lungs obtained after cardiac death. Eur J Cardiothorac Surg. 48(4):542-547.

Hytting-Andreasen R, Balk-Møller E, Hartmann B, et al. 2018. Endogenous glucagon-like peptide- 1 and 2 are essential for regeneration after acute intestinal injury in mice. PLoS ONE. 13(6):e0198046.

Inci I. 2017. Donors after cardiocirculatory death and lung transplantation. J Thorac Dis. 9(8):2660-2669.

Kootstra G, Daemen JHC, Oomen APA. 1995. Categories of non-heartbeating donors. Transpl Proc. (27):2893-2894.

Machuca TN, Cypel M, Keshavjee S. 2013. Advances in lung preservation. Surg Clin North Am. 93(6):1373-1394.

Nilsson T, Wallinder A, Henriksen I. 2019. Lung transplantation after ex vivo lung perfusion in two Scandinavian centres. Eur J Cardiothorac Surg. 1;55(4):766-772.

Porteous MK, Lee JC. 2017. Primary Graft Dysfunction After Lung Transplantation. Clin Chest Med. 38(4):641-654.

Raemdonck DEM Van, Jannis NCP, Leyn PRJ De, et al. 1998. Warm lschemic Tolerance in Collapsed Pulmonary Grafts Is Limited to 1 Hour. Ann Thorac Surg. 228(6):788-796.

Spratt JR, Mattison LM, Iaizzo PA, et al. 2017. An experimental study of the recovery of injured porcine lungs with prolonged normothermic cellular ex vivo lung perfusion following donation after circulatory death. Transpl Int. 30(9):932-944.

Spratt JR, Mattison LM, Iaizzo PA, et al. 2018. Lung transplant after prolonged ex vivo lung perfusion: predictors of allograft function in swine. Transpl Int. 31(12):1405-1417.

van Suylen V, Luijk B, Hoek RAS, et al. 2017. A Multicenter Study on Long-Term Outcomes After Lung Transplantation Comparing Donation After Circulatory Death and Donation After Brain Death. Am J Transpl. 17(10):2679-2686. 